Winifred Davies (Aberystwyth)/Nils Langer (Bristol)

\title{
Die Sprachnormfrage im Deutschunterricht: das Dilemma der Lehrenden*
}

\begin{abstract}
Üblicherweise wird behauptet und erwartet, dass für den Deutschunterricht die deutsche Standardsprache zumindest als Zielsprache, wenn nicht gar als Unterrichtssprache gilt. Die Forschungen der germanistischen Soziolinguistik und Sprachlehrforschung zeigen allerdings, dass keinesfalls Einigkeit darüber besteht, was denn , die deutsche Standardsprache überhaupt sei, ob, und wenn ja, wie viel Variation sie beinhaltet, und wie mit Normabweichungen seitens der Schüler/innen umzugehen sei.

Unser Beitrag beschäftigt sich mit der Rolle der Deutschlehrenden - sowohl an deutschsprachigen Schulen als auch im Rahmen des DaF-Unterrichts an britischen Hochschulen - um zu erörtern, welche Erwartungen sie an die sprachliche Normenkonformität ihrer Schüler/innen haben und welche praktischen Probleme ihnen hierbei begegnen. Unterstützt durch historische Belege aus dem Schulalltag im 19. Jahrhundert, diskutieren wir Kontinuitäten und Innovationen in der Selbsteinschätzung von Deutsch- und DaF-Lehrer/innen zu ihrer Rolle als Sprachnormvermittler/innen und stellen die Frage, wie groß ihre Rolle tatsächlich ist.
\end{abstract}

\section{Einleitung}

Dass die Schule für den Erwerb der Standardsprache besonders wichtig ist, wird allgemein für selbstverständlich gehalten. Im Schulunterricht sollte - so zumindest die vorherrschende Meinung der Öffentlichkeit - besonders darauf geachtet werden, eine normenkonforme Sprache in Wort und Schrift zu vermitteln, um den Schüler/innen zu ermöglichen, am gesellschaftlichen Leben teilzunehmen und nicht später als Erwachsene durch Leseschwächen, schlechte Rechtschreibung oder falsche Kasusrektion benachteiligt zu werden. Eine entregionalisierte Grammatik, eine akzentfreie Aussprache und ein reicher Wortschatz, gerne mit Latinismen und Graezismen - nicht aber Anglizismen - angereichert, gilt immer noch als Idealvorstellung vieler Teile der Bevölkerung ${ }^{1}$ - auch wenn die Meinungen darüber, was denn akzentfrei ${ }^{2}$ oder was entregionalisiert ist, in der Praxis weit auseinander gehen.

* Wir danken dem Publikum der IDS-Jahrestagung 2013 für seine hilfreichen Anmerkungen und Kim Pätzold (Bristol) für ihre Hilfe beim Korrekturlesen.

1 Wobei es nicht wirklich klar ist, ob dies die Meinung der Mehrheit der Bevölkerung insgesamt oder nur der Mehrheit der sich zu dieser Problematik äußernden Bevölkerung widerspiegelt.

2 Dass es möglich ist, ohne Akzent zu sprechen, ist natürlich ein Mythos (vgl. Lippi-Green 2012, S. 44 f.). 
Ist ,Norddeutsch' ein regionaler Akzent oder die anzustrebende Idealaussprache? Darf man ,Da kann ich nichts für' sagen oder gar schreiben? Ist wegen + Dativ immer unakzeptabel, oder gibt es Ausnahmen? Und ist ,Der Tag, wo wir ankamen' Teil der Standardnorm? ${ }^{3}$

In diesem Aufsatz beschäftigen wir uns mit der Rolle der Lehrenden bei der Vermittlung der Standardsprache und ihrem Dilemma, dass sie es irgendwie niemandem recht machen können. Es wird ihnen regelmäßig vorgeworfen, sie wären nicht streng genug, was sprachliche Standards angeht, und würden ihre Aufgaben nicht richtig erfüllen: Wolf Schneider, zum Beispiel, Verfasser vieler laienlinguistischer Sprachratgeber und ehemaliger Leiter der Hamburger Journalistenschule, wirft ihnen vor, ,sie würden ihre historische Pflicht gegenüber der Sprache nicht mehr erfüllen wollen" (Schneider 2001, S. 216) und würden eine Spaßpädagogik treiben, die den Schülern jede Mühe erspart (Zeit online, 11.5.2012).

Weiterhin hat in Deutschland und in der Schweiz der sogenannte PISASchock dafür gesorgt, dass die Kompetenzen von Lehrenden noch schärfer unter die Lupe genommen werden. Ihnen wird auferlegt, die sprachlichen Kompetenzen der Schüler/innen zu verbessern, aber gern werden in dieser Diskussion die Leistungen von Schulen mit sehr unterschiedlichen Sozialprofilen recht wahllos angeführt, um sprachapokalyptische Szenarien auszumalen. Was sprachliche Kompetenzen tatsächlich sind, hängt eben nicht nur von den Grundvoraussetzungen ab, z.B. der Familiensprache, sondern auch davon, was man als erstrebenswert ansieht: Statt lobend zu beobachten, wie die Jugend durch ihre multimedialen Zugänge zu Texten eine Reihe von Registern zu verschriftlichen lernt, anstatt sich eigentlich nur in der formellen Hochsprache ausdrücken zu können, empfinden viele Kommentatoren dies im Gegenteil als Chaos oder Verwirrung. Diese Reaktion findet man auch häufig angesichts der weit verbreiteten Mehrsprachigkeit an den Schulen, obwohl die Überzeugung, dass die Schulklassen früher homogener waren, sicher nicht der Wirklichkeit entspricht.

In den Lehrplänen und amtlichen Richtlinien finden sich häufige Bezüge zur Standardsprache als einzig akzeptabler Unterrichtssprache. Zwar heißt es oft, Schüler/innen sollten ,situations- und adressatengerecht sprechen und schreiben" (Kernlehrplan für den verkürzten Bildungsgang des Gymnasiums Sekundarstufe I (G8) in Nordrhein-Westfalen. Deutsch 2007, S. 11), und in ihrem Sprachgebrauch eine ,reale[...] Differenzierung der Sprache und des Sprachverhaltens" (Richtlinien für die gymnasiale Oberstufe Nordrhein-Westfalen, zitiert in Volmert 1997, S. 69) aufweisen, aber genauso wird

In Davies (2000) und Davies/Langer (2006) werden Beispiele für divergierende Meinungen von Sprachnormautoritäten (Deutschlehrenden) und angesehenen Nachschlagewerken (Duden-Bänden) beschrieben. Beim temporalen wo wurde klar, dass laienlinguistische Sprachratgeber (z.B. Illgner 2001) eher die Meinung der Sprachnormautoritäten wiedergeben als Werke mit höheren wissenschaftlichen Ansprüchen. 
verlangt, dass „für die Bewertung der Darstellungsleistung“ auf die „Berücksichtigung standardsprachlicher Normen" besonders Gewicht gelegt werde (Richtlinien und Lehrpläne für die Sekundarstufe II Gymnasium/Gesamtschule in Nordrhein-Westfalen 1999, S. 67). Ganz deutlich findet man diese Position in den Beschlüssen der Kultusministerkonferenz (KMK) von 2003: „Die Schülerinnen und Schüler bewältigen kommunikative Situationen in persönlichen, beruflichen und öffentlichen Zusammenhängen situationsangemessen und adressatengerecht. Sie benutzen die Standardsprache."

Während des Universitätsstudiums werden angehende Lehrer/innen wenn sie überhaupt soziolinguistische Seminare belegen - zwar von der Differenztheorie und der Äquivalenz sprachlicher Varietäten lernen, aber die Tatsache, dass ein Buch wie Sick (2004) in einer DaF-Fachzeitschrift positiv rezensiert und für den Lehrbetrieb wärmstens empfohlen wird, weil dort darin doch endlich mal genau gesagt werde, was richtig und was falsch ist, zeigt, dass soziolinguistische Theorie und tatsächliche Praktiken im Unterricht keinesfalls gleichzusetzen sind. ${ }^{4}$ Ist es überhaupt möglich, die Belange der Lehrenden, die oft ganz konkrete Kommunikationsprobleme schnell lösen wollen und schriftliche und mündliche Arbeiten transparent und fair benoten müssen, mit der Perspektive von Soziolinguist/innen als Verfechter/innen eines deskriptiven, variationstoleranten Ansatzes in Einklang zu bringen? In diesem Beitrag reflektieren wir über dieses Dilemma der Lehrenden, indem wir Theorie - in der Form von Grammatikeinträgen, Lehrplänen, aber auch metasprachlichen Diskussionen - und Praxis - soweit diese für uns zugänglich sein können - miteinander vergleichen. Wir sprechen hier nicht nur von unserer Warte als germanistische Soziolinguist/innen aus, sondern auch als praktizierende DaF-Lehrende an britischen Hochschulen. In unserer Forschung beschäftigen wir uns mit theoretischen Aspekten von Standardisierungsprozessen, mit Ideologien der Standardsprache, mit Kritiken der Theorie der Situationsadäquatheit usw., aber ein Teil unserer Arbeit - wie bei vielen Germanistikdozent/innen im Ausland - besteht darin, Sprachunterricht zu geben und sprachpraktische Übungen zu korrigieren, was bedeutet, dass wir jede Woche selbst versuchen müssen, beide Perspektiven unter einen Hut zu bringen.

Ziel des Aufsatzes ist, die Probleme der Sprachlehrenden aufzuzeigen, denn der Begriff Standardsprache (bzw. Hochsprache oder Hochdeutsch) wird in den Lehrplänen und Lehrmaterialien fast immer ohne nähere Spezifizierung und als nicht weiter erklärungsbedürftig verwendet; in der Praxis aber muss er ganz unterschiedliche Sprachvarianten umfassen, wenn er sich nicht als unbrauchbar erweisen soll (siehe Eichinger 2005, S. 363).

Man vgl. allerdings hierzu, welche heftigen Reaktionen seitens der Soziolinguistik auf diese Rezension folgten (z.B. Maitz/Elspaß 2007, auch 2011). 


\section{Lehrer/innen und Stigmatisierung sprachlicher Konstruktionen - historische Dimension}

Wie oben angedeutet, schreiben wir den Lehrer/innen, speziell den Deutschlehrer/innen, eine besondere Rolle als Normautoritäten (Ammon 1995) und Normvermittler/innen zu. Man könnte sie eventuell auch als Normgeber betrachten: Peter Braun zum Beispiel beobachtete in einer empirischen Untersuchung, dass morphologische und orthographische Varianten, die laut Duden alle gültig seien (z. B. ,des Tales' und ,des Tals), in der Schule nicht immer berücksichtigt würden (Siegl 1986, S. 86), sondern dass sich in der Praxis die Lehrer/innen für nur eine Variante entschieden und diese als die einzig richtige vermittelten. Daraus folgt, dass, selbst wenn Lehrer/innen nicht direkt am Kodifizierungsprozess teilnehmen, sie dennoch durch ihre jeweilige Unterrichtsspraxis die womöglich viel ausschlaggebendere Macht darüber haben, was als standardsprachlich wahrgenommen wird.

Es stellt sich die Frage, ob sie diese Funktion auch schon früher, also zu den Anfängen der Standardisierung des Deutschen hatten. Die Rolle des Sprachunterrichts und der Grammatikschreibung waren schon von Anfang an verbunden. Die ersten relativ vollständigen Grammatiken des Deutschen aus den 1570er Jahren waren Fremdsprachengrammatiken und ihre Autoren somit DaF-Lehrer. Zielsprache war ganz allgemein ,Deutsch', auch wenn die verschiedenen Autoren damit recht unterschiedliche Varietäten meinten. ${ }^{5} \mathrm{Ob}$ mit dieser Zielsprache auch explizites Prestige verbunden war, ist unklar. Es bestehen leider kaum zeitgenössische metasprachliche Kommentare über die Praxis des Unterrichts - weder für $\mathrm{DaF}$ noch für den muttersprachlichen Unterricht (vgl. Müller 1882). Wir sind für diese Zeit also in der Regel darauf angewiesen, aus gedruckten Quellen, z.B. Vorworten von Grammatiken und sprachlichen Beschreibungen in solchen Werken, Rückschlüsse auf die soziolinguistische Wirklichkeit zu schließen. Für das 17. Jahrhundert gibt es eine Fülle an Forschungsliteratur, und gerade die Rolle der Grammatiker ist auch in jüngster Zeit detailliert erforscht worden (siehe Gardt 1999; Hundt 2000; Langer 2001; McLelland 2012). Die Effektivität der präskriptiven Grammatiken für den Sprachgebrauch überzeugend zu messen, bleibt allerdings schwierig. Die erste deutschsprachige Grammatik (Kromayer 1618) dient der Lehre der Grammatica im Allgemeinen - für deutsche Muttersprachler -, nicht der deutschen Grammatik im Speziellen, und sie ist somit keineswegs eine vollständige Beschreibung einer Varietät des Deutschen, sondern nur eine lateinische Grammatik mit deutschen Beispielen. Nicht der Unterricht der deutschen Sprache war das Ziel, sondern Kenntnisse der grammatikalischen Fachbegriffe, damit anschließend das

Vgl. Langer (2004) zu morphosyntaktischen Unterschieden in der DaF-Zielsprache der Frühen Neuzeit. 
Lateinische gelernt werden konnte. Dessen ungeachtet entsteht in dieser Zeit ein großes Interesse an der Hebung des Deutschen, dessen Variantenvielfalt durch Spracharbeit (siehe Hundt 2000) gebändigt werden müsse, um eine ausgebaute und würdige Standardsprache zu erreichen. Zahlreiche Grammatiken beschäftigen sich mit dieser Aufgabe, wobei für die Erforschung der Standardsprache nicht nur interessant ist, welche Konstruktionen für die Prestigevarietät ausgewählt wurden, sondern auch, welche ausgeschlossen blieben (siehe Davies/Langer 2006).

Aber erst für das 18. Jahrhundert erhalten wir einen besseren Einblick darüber, welche Sprachnormen im Schulalltag vermittelt wurden, da die Schulbildung auf breitere Schichten ausgeweitet wurde und Lese- und Lehrbücher für den Schulgebrauch veröffentlicht wurden. Die Autoren dieser Werke beziehen klare, wenn auch nicht vollständige, Positionen zu Konstruktionen, die nicht zu gebrauchen seien. Zur Polynegation im Deutschen schreibt Hemmer (1769, S. 188 f.), dass ,[n]icht allein der gemeine Mann, sondern auch eine gute Menge vornehmer Leute" diese oft benutzten, sie aber dennoch überflüssig sei. Heynatz, in der „Deutschen Sprachlehre zum Gebrauch für die Schulen" (1777, S. 279), bezeichnet diese Konstruktion noch expliziter als unrichtig, und für Adelung (1793, S. 484) klingt sie ,in der anständigen Schreibart [...] überaus widrig“", auch wenn man sie doch oft höre und lese. In der Standardisierungsgeschichte ging es also nicht nur darum, zu benennen, was zur Standardsprache gehört, sondern auch, was nicht dazu gehört. Mit der Entstehung der Standardsprache gab es nun eine erstrebenswerte Sprachnorm, die zur Ausbildung der aufstrebenden Bevölkerungsschichten gehörte, und die - wie die Schulgrammatik von Heynatz (1777) zeigt - auch Unterrichtsgegenstand war. Diese Schulbücher bezogen eine explizite Stellung in der Normierung der Zielsprache.

Doch gilt dies auch für die Volksschulen, die seit dem späten 18. Jahrhundert immer weiter verbreitet waren? Inwiefern waren Lehrer als Gruppe an der Ausformung und Verbreitung der entstandenen und entstehenden Standardsprache beteiligt? Was können uns die historischen Quellen über den Sprachgebrauch im Unterricht und das metalinguistische Verständnis der Lehrer mitteilen?

\section{Schulunterricht und Sprachunterricht}

Seit dem späten 18. Jahrhundert finden sich immer mehr Belege dafür, dass Schullehrer ein aktives Interesse am Muttersprachunterricht nahmen - sowohl im Rahmen der weiter voranschreitenden, allgemeinen Standardsprachideologie wie auch als Teil der erstrebten Nationalerziehung. Hier müssen wir allerdings ein wenig differenzieren. Die Gelehrten- oder Lateinschulen beschäftigten sich erst recht spät mit dem Muttersprachunterricht als 
selbstständigem Fach und konzentrierten sich zu Anfang des 19. Jahrhunderts noch lange Zeit auf die Vermittlung der altphilologischen Sprachen (G. Schmidt 1985, S. 252). Da im zeitgenössischen Denken zur Vermittlung der neuen, als „gemeinsam empfundenen Nationalliteratur“ (ebd.) eine gefestigte grammatische Norm nötig war, wurden die von Adelung proponierten Standards im Unterricht eingeführt. Im Laufe des 19. Jahrhunderts melden sich auch Lehrer immer wieder mit Beiträgen - ab 1885 vor allem im Rahmen des Allgemeinen Deutschen Sprachvereins (ADSV) - zu korrektem Sprachgebrauch für die Schule (siehe Durrell 2007; Durrell in diesem Bd.; Ziegler 1998). Intensive Debatten finden sich auch unter Grammatik- und Lehrbuchschreibern, teils in den jeweiligen Vorworten, teils als Kommentar zu bestimmten Konstruktionen in den jeweiligen Werken, teils aber auch in Verbandszeitschriften für Pädagogen (siehe Davies/Langer 2006; Durrell 2007; Law 2007).

Für die Volksschulen gilt auf der anderen Seite, dass höhere Lernziele nur selten verfolgt wurden (noch weniger nach 1848/49, siehe Bluhm-Faust 2005, S. $107^{6}$ ), was aber auch stark von den einzelnen Lehrern abhing. Unter den Volksschullehrern finden sich immer einige, die aktiv in die metasprachlichen Debatten zur Standardisierung eingreifen (siehe H. Schmidt 1985). Hierzu gehört neben dem Themenkomplex der exakten grammatikalischen Wertung von Sprachvarianten auch die Diskussion zur Stellung des Dialekts in der Schule.

\section{Dialekt in der Schule}

Wir führen hierzu exemplarisch Beispiele aus dem norddeutschen $\operatorname{Raum}^{7}$ an, wo sich zu diesem Thema zwei Positionen finden: zum einen die absolute Ablehnung des hiesigen Dialekts, des Niederdeutschen, da dies bäurisch klinge und ein Hindernis für die Bildung der Landbevölkerung darstelle (siehe Wienbarg 1834), sowie womöglich auch als anti-national interpretiert werden könne, da die Sprache der nationalen Einigung allein das Hochdeutsche sei. Zum anderen finden sich aber auch zahlreiche Stimmen, die sich sehr stark für das Niederdeutsche einsetzen, sowohl aus ideologischen Gründen (also das Niederdeutsche als die historische und traditionsträchtige Sprache

„Für den elementaren Deutschunterricht [in Baden, W.D./N.L.] bedeutete dies, dass nun von der Vermittlung der deutschen Standardsprache und vor allem von der Denkschulung abgesehen wurde“ (Bluhm-Faust 2005, S. 107).

7 In Bluhm-Faust (2005, S. 546) lesen wir, dass es auch andernorts Diskussionen zu diesem Thema gab. Hildebrand (1867) und von Raumer (1852) schlugen vor, den Unterricht in der Standardsprache an der Mundart der Schüler anzuknüpfen, d.h. die Mundart durfte auch im Klassenzimmer vorkommen. Es gab aber auch „Stimmen, die die Mundart ganz aus der Schule verbannen wollten“ (Bluhm-Faust 2005, S. 546). 
der Norddeutschen) wie auch aus pädagogischen Gründen (also das Niederdeutsche als die Muttersprache der eingeschulten Kinder; siehe Langer/ Langhanke 2013). Im Folgenden zeigen wir einige Beispiele für die besonderen soziolinguistischen Bedingungen in Schleswig-Holstein. In den Kirchen- und Schulinspektionsberichten aus Schleswig-Holstein finden sich auch immer wieder Kommentare, die uns einen Einblick in das negative Stigma der niederdeutschen Sprache geben: ${ }^{8}$

In dieser mangelhaften Bildung hat es denn auch seinen Grund, daß die plattdeutsche Sprache hier viel mehr als an andern Orten Umgangssprache ist, welches für die Kinder den Nachtheil hat, daß einige bei ihrer Aufnahme in unsere Schule kein hochdeutsches Wort sprechen können. (Kappeln (Angeln), 1843)

Doch nicht nur das Plattdeutsch der Kinder wird kritisiert. In den Berichten der Schulinspektoren finden sich auch Anmerkungen über das fehlerhafte Deutsch des Lehrers bzw. dessen Verwendung des Niederdeutschen im Unterricht, die uns Aufschlüsse über die tatsächliche Unterrichtssprache geben:

Meine hieselbst übernommene Schule [c. 1848] befand sich in der traurigsten Verfassung. Selbst die größeren Kinder konnten nicht einmal richtig buchstabieren, vielweniger [sic!] lesen. Der alte Lehrer hatte in der Schule nur plattdeutsch gesprochen. (Vaale (Dithmarschen), 1851)

In seiner Kritik der Kieler Schulen von 1907 und 1910 bemängelt der Schulinspektor die fehlerhafte (d.h. norddeutsche!) Aussprache des Lehrers im Unterricht:

Kiel-Gaarden, 1907, XIII. Knaben-Volksschule

Klasse VIII [...] Gelesen wird recht fliessend, aber auf Betonung und lautreine Aussprache, namentlich das Z - es ist überhaupt erstaunlich, wie wenig Lehrer ein deutliches Z sprechen können und den Unterschied zwischen Z und S hören muss mehr Wert gelegt werden. Desgleichen auf den Wohlklang der Sprache. Der Lehrer selbst spricht ziemlich bäurisch.

Kiel-Knooper Weg, 1910, V. Mädchen-Volksschule

Klasse VI b [...] Die Kinder rechnen mündlich und schriftlich befriedigend, schreiben die Zahlen gut, sprechen aber mässig: „ach ma vie is swei und dreisig““

Eine ähnliche Bewertung nicht-hochdeutscher Sprachen findet sich auch in der Intoleranz gegenüber den autochthonen Minderheitensprachen Friesisch, Südjütisch und Reichsdänisch. Im 19. Jahrhundert klagen die Volksschullehrer in vielen Kirchspielen des heutigen deutschdänischen Grenzlands immer wieder über die Kinder mit friesischer oder dänischer Muttersprache, die aus diesem Grund nur über unzureichende Kenntnisse der hochdeutschen Unterrichtssprache verfügen (vgl. Langer 2011):

„Hindernisse: 1, der Umstand, daß die Umgangssprache hier platt dänisch ist, während der Unterricht in deutscher Sprache ertheilt wird.“ (Aventoft, 1847);

„Wie für den Unterricht überhaupt die friesische Muttersprache unserer Kinder ein großes und nicht zu beseitigendes Hinderniß ist, indem den Kindern bei ihrem Eintritt in die Schule die deutsche Sprache ihnen völlig fremd ist, so dass sie erst nach mehreren Jahren den Lehrer verstehen.“ (Horsbüll, 1872). 
Diese Belege zeigen, dass die Aufgabe der Volksschullehrer sehr wohl die Lehre - und der eigene Gebrauch - des korrekten Hochdeutsch war und dass, wo dies nicht zufriedenstellend geschah, dies bemerkt und kommentiert wurde. Das Niederdeutsche wurde jedoch nicht von allen als für den Schulunterricht ungeeignet stigmatisiert. Womöglich durch seine besondere Stellung als Sprachvarietät mit langer, eigenständiger Geschichte (Hansesprache, Bibelübersetzung) genoss und genießt das Niederdeutsche einen besonderen Status, der ihn heutzutage zur einzigen ,offiziellen' Regionalsprache in Deutschland gemacht hat. Dieser besondere Status hatte bereits in den Diskussionen des 19. Jahrhunderts seine Auswirkungen, und zwar eben auch unter den Volksschullehrern. In den Lehrervereinigungen wurde mindestens seit den 1840er Jahren diskutiert, ob und inwiefern das Niederdeutsche Eingang in den Schulunterricht finden möge, und zwar nicht primär deshalb, weil es den Kindern den Lernerfolg erleichtern würde, sondern weil das Niederdeutsche die angestammte Sprache der Norddeutschen sei. Diese Diskussion fand in verschiedenen Foren statt, unter anderem auch in der Schleswig-Holsteinischen Schulzeitung (SHSZ), dem Vereinsblatt der Volksschullehrer. Hier lesen wir 1855 über die Mundart:

SHSZ No 18, 3.2.1855, S. 70 f., Zur Bücheranzeige, von F. Dunker

Wenn nun das Volk dabei bleibt, in der Mundart zu denken, so ist im Laufe der Zeit ein Zwiespalt entstanden zwischen Cultur und Literatur. Dieser Zwiespalt läßt sich nur heben, wenn die Volksschule es übernimmt, zwischen Dialect und Schriftsprache, zwischen Cultur und Literatur, zwischen Volksthum und Schriftthum zu vermitteln. [...]

$[N]$ ur durch eine pädagogische Anleitung zum Uebertragen der eigenen, plattdeutschen Gedanken in die Formen der Schriftsprache werden unsere Schüler die nöthige Gewandheit und Sicherheit erwerben, um sich später im Leben der Schriftsprache mit Anstand bedienen zu können.

Das Niederdeutsche findet starke Unterstützung durch ihre Neuverschriftung durch Klaus Groth (1852) und wird auch in einigen Lesebüchern für Volksschulen zumindest als Hilfsmittel zu einem erfolgreicheren Erwerb der Hochsprache berücksichtigt (z.B. Burgwardt 1867; Knees (Hg.) 1878; siehe www.spsh.uni-kiel.de/Archiv/sprachen-der-schule/schulbuecher). Es bleibt jedoch unklar, inwieweit das Niederdeutsche tatsächlich im Unterricht verwendet wurde, um den Kindern den Zugang zum Lehrstoff zu erleichtern. Sicherlich werden einige Lehrer niederdeutsch gesprochen haben oder zumindest als Hilfsmittel verwendet haben - dies zeigen zahlreiche Anekdoten aus Lebenserinnerungen und aus den Beiträgen in der Schleswig-Holsteinischen Schulzeitung. Aber selbst von den entschiedensten Verfechtern des Niederdeutschen wurde stets betont, dass die nationale Einigung Deutschlands in der hochdeutschen Sprache zu geschehen habe.

Was wir an diesen Beispielen sehen, ist, dass sich Lehrer auch im 19. Jahrhundert mit Sprachenfragen beschäftigt haben; allerdings weniger damit, 
welche speziellen Varianten der Standardsprache der Zielsprache entsprechen, als vielmehr welche Funktion - wenn überhaupt eine - den Dialekten bzw. der Nicht-Hochsprache eingeräumt werden möge und welche Modelle nachgeahmt werden sollten.

Nicht nur im norddeutschen Raum, sondern im ganzen Deutschland des 19. Jahrhunderts beteiligen sich Lehrer an der Diskussion über die korrekte Form des Hochdeutschen. Bluhm-Faust (2005, S. 540) konstatiert, „dass sich die Lehrer ihrer besonderen Rolle bei der Vermittlung der deutschen Standardsprache bewusst waren“. Als Quellen liegen uns zu diesem Thema neben Lehrbüchern und Sprachratgebern vor allem die vielen Beiträge von Lehrern in einschlägigen Vereinszeitschriften und Zeitungen vor. ${ }^{9}$ Auch wenn „,[d]ie Standardvarietät Deutsch Ende des 19. Jahrhunderts als vollkommen konstituiert [gilt]“ (Schneider-Mizony 2008, S. 396), weist die hohe Anzahl von Sprachratgebern auf dem Markt doch darauf hin, dass es noch relativ viele grammatische Grauzonen gab. Wustmanns „Sprachdummheiten" erschien zwischen 1891 und 1966 in 14 Auflagen, und Hirt (1925, S. 239) berichtet, dass jedes Heft der „Zeitschrift des Allgemeinen Deutschen Sprachvereins“" (1886-1924) viele Fragen zu konkurrierenden Formen und Zweifelsfällen enthalte. Laut H. Schmidt (1985, S. 175) fühlten sich viele Lehrer von den historisch und deskriptiv orientierten wissenschaftlichen Grammatiken der Zeit im Stich gelassen, und Hirt (1925, S. 242) behauptet, die Lehrer hätten sich an Werke wie die von Wustmann wenden müssen, weil sie auf der Hochschule kaum etwas über Sprachrichtigkeit erfahren hätten.

Was die Lehrpläne angeht, war schon „,[a]m Anfang des 19. Jahrhunderts [...] der richtige Gebrauch der hochdeutschen Sprachform erklärtes Ziel des deutschen Sprachunterrichts", zumindest für die Volksschulen in Baden (Bluhm-Faust 2005, S. 543). Naumanns Behauptung (1986, S. 93), dass

die normative Vermittlung jeweiliger Gegenwartssprache [...] zu allen Zeiten das oberste Lernziel des deutschen Sprachunterrichts [war und ist]. Alle Schüler sollten von jeher gut und richtig reden, schreiben und lesen lernen[,]

scheint uns plausibel, auch wenn es beizeiten immer wieder kritische Stimmen gegeben hat, die für die stärkere Berücksichtigung der tatsächlichen Muttersprache der Kinder, also der Dialekte, beim Erlernen des Hochdeutschen plädierten (von Raumer und Hildebrand gehören dazu, siehe BluhmFaust 2005, S. 132). Unbestritten war die Rolle der Hochsprache für das Konzept der Sprach-, Kultur- und Staatsnation (Ziegler 1998); was das aber konkret für die angestrebte Variationslosigkeit (und die Realität der Variation auch in der Hochsprache) bedeutete, ist deutlich schwieriger zu ermessen.

Es bleibt allerdings unklar, ob dies wirklich für eine Mehrheit der Lehrer galt oder nur für wenige, sehr aktive, Korrespondenten. Für Schleswig-Holstein kann man zum Beispiel sagen, dass, auch wenn die SHSZ wohl von fast allen Volksschullehrern der Region bezogen und gelesen wurde, die namentlich genannten Beiträger zu den Artikeln insgesamt recht wenige sind. 
Nachdem Adelung 1782 die Frage „Was ist Hochdeutsch?“ mit dem meißnischen Deutsch der höheren Klassen beantwortet hatte und die Rolle der besten Schriftsteller einzig auf die Rolle von Multiplikatoren reduziert wurde, änderte sich diese Position im Laufe des 19. Jahrhunderts. Als Idealnorm galt dann lange Zeit - und für viele noch heute! - die klassische Literatursprache, also die Sprache Goethes und Schillers. Mit der Ausweitung der Standardsprache auf weitere Kommunikationsbereiche im Laufe des 19. Jahrhunderts kam auch die größere Akzeptanz der Literatursprache als Vorbild für die Sprachnorm, was sich in vielen sprachkritischen Schriften widerspiegelte (Ziegler 1998). ${ }^{10}$ Die sprachkritischen Diskussionen, die sich nicht nur in ideologischen Bahnen auf allgemeine Normvorstellungen (Rolle bestimmter Regionen, Berufsgruppen, Institutionen) und rhetorische Figuren der Dichtersprache, sondern eben auch auf konkrete morphosyntaktische Konstruktionen bezogen (siehe Davies/Langer 2006), sagen allerdings wenig über die tatsächlich verwendete Varietät und verwendeten Sprachvarianten im Schulunterricht aus. Die Aussage, dass das Hochdeutsche im Unterricht zu verwenden sei, macht keine Aussage darüber, was tatsächlich unter Hochdeutsch verstanden wurde, und es ist kaum wahrscheinlich, dass im 19. Jahrhundert weniger Variabilität in der Hochsprache zu finden war, als dies noch heute der Fall ist.

Dieser Überblick über die Beziehung von Sprache und Schule soll gezeigt haben, dass Lehrende sehr wohl eine Rolle als Normvermittler im Muttersprachunterricht gespielt haben, dass es aber mit den historischen Quellen nur selten möglich ist, etwas über den tatsächlichen Sprachgebrauch im Schulalltag zu ermitteln. Noch 1925 schrieb Herman Hirt (1925, S. 240): „Wir haben durchschnittlich mit der Tatsache zu rechnen, daß die neuhochdeutsche Schriftsprache erst auf der Schule erlernt werden muß“". Wie dies genau geschehen ist - durch die Stigmatisierung bzw. Nichtverwendung von nichtstandardsprachlichen Varianten - und inwiefern dies auch für die Volksschule gegolten haben wird, ist zum großen Teil noch nicht ermittelbar.

Kommen wir also nun zurück in die Gegenwart und diskutieren wir, welche Vorstellungen von muttersprachlicher Sprachrichtigkeit heutzutage in Lehrplänen und Unterricht zu finden sind.

\section{Erwartungen an Lehrende: Umfragen und Lehrpläne}

Die Erwartung, dass Lehrkräfte eine besondere Rolle bei der Vermittlung der institutionalisierten Normen der deutschen Sprache spielen, kommt in

10 „So besteht für viele Zeitgenossen wie Keller, Lehmann, Matthias, v. Wolzogen u.a. kein Zweifel mehr daran, daß sich das, gute und richtige Deutsch“ vorrangig bei den Klassikern Goethe und Schiller findet“" (Ziegler 1998, S. 29). 
verschiedenen Umfragen zum Ausdruck (z.B. Stickel/Volz 1999; Eichinger et al. 2009). So waren im Jahre 1999 83,6\% der Befragten der Meinung, dass die Schule sich um die zukünftige Entwicklung der deutschen Sprache kümmern sollte. Für Mannheim-Neckarau fand Davies (1995), dass viele Dialektsprecher/innen es nach eigener Einschätzung fast ausschließlich der Schule überlassen würden, ihren Kindern Kompetenz in der Standardsprache beizubringen.

Man braucht nur einen kurzen Blick in die Lehrpläne für das Fach Deutsch an den Schulen zu werfen, um festzustellen, dass das von Naumann beschriebene Ziel immer noch gilt ${ }^{11}$ so z.B. die von der KMK für den Mittleren Bildungsabschluss geforderten Bildungsstandards (2003), die schon oben zitiert wurden: „Die Schülerinnen und Schüler bewältigen kommunikative Situationen in persönlichen, beruflichen und öffentlichen $\mathrm{Zu}$ sammenhängen situationsangemessen und adressatengerecht. Sie benutzen die Standardsprache“. In den Rahmenrichtlinien Deutsch für das Gymnasium Schuljahrgänge 5-12 in Sachsen-Anhalt (2003) lesen wir, dass „,[d]ie in den Schuljahrgängen 1-4 der Grundschule vermittelten Kenntnisse, Fähigkeiten und Fertigkeiten im Gebrauch der deutschen Standardsprache [...] weiterentwickelt und ausgebaut" werden (S. 6). Im Lehrplan Deutsch für das Gymnasium in Sachsen (2004) „,steht die Hochsprache in ihrer schriftlichen Form als die am weitesten tragende Varietät der deutschen Sprache“ im Mittelpunkt des Unterrichts“ (S. 2). Im Rahmenplan Deutsch für das neunstufige Gymnasium in Hamburg (2003, S. 5) lesen wir:

Der Deutschunterricht entfaltet in vier Arbeitsbereichen die sprachliche Ausdrucksfähigkeit der Kinder und Jugendlichen, damit sie zunehmend sicher in der sprachlichen Bewältigung privater, beruflicher und öffentlich-gesellschaftlicher Lebenssituationen werden. Die Verwendung der hochdeutschen Allgemeinsprache dient diesem Ziel.

Es wird in diesen Richtlinien also klar davon ausgegangen, dass es die Standard- oder Hochsprache gibt und dass man sich einig ist, was damit gemeint sei.

Untersuchungen aus Süddeutschland zeigen, dass die Deutschlehrer/ innen die Rolle der Schule bei der Vermittlung einer standardsprachlichen Norm als selbstverständlich ansehen und die Erwünschtheit einer Standardvarietät nicht in Frage stellen (z.B. Davies 2000; siehe Wagner 2009 für ähnliche Ergebnisse für Trier und Umgebung). Von daher entspricht das berufliche Selbstverständnis der Lehrkräfte den Erwartungen von Eltern, Bildungspolitikern und Teilen der Öffentlichkeit. Die Frage stellt sich allerdings, ob die angestrebte und vorausgesetzte Einigkeit über die tatsächliche Form der Standardsprache auch in der Praxis so vorhanden ist, obwohl

$11 \quad$ Nicht nur in Deutschland: In Zürich z.B. wird „D[ie] Pflege der Standardsprache als Grundlage der Verständigung aller Deutschsprechenden" als ein Ziel des Deutschunterrichts vorgeschrieben, siehe www.kzo.ch/index.php?id=715 (Stand: 27.9.2010). 
Deutsch (vor allem die bundesdeutsche Variante) einen umfangreichen Kodex besitzt und alle Sprachebenen kodifiziert sind. ${ }^{12}$ Trotzdem gestaltet sich das Identifizieren von allgemein akzeptierten Regelwerken und Modellen schwieriger, als man vielleicht annehmen würde (siehe auch den Beitrag von Klein in diesem Band). Davies (2000) zeigt, dass es innerhalb einer Gruppe von Realschullehrer/innen in der Mannheimer Gegend relativ wenig Einigkeit darüber gab, in welchen Werken die Regeln für Standarddeutsch zu finden seien. Sie wurden gefragt, was sie unter Standarddeutsch verstehen würden und wo es ihrer Meinung nach zu finden sei. Die Duden-Bände wurden am häufigsten erwähnt, aber auch nur von knapp über der Hälfte der Lehrer (54,5\%). In einer zweiten Untersuchung, diesmal mit Gymnasiallehrer/innen (Davies 2005) in Süddeutschland, waren die Antworten einheitlicher, und 92\% meinten, die Regeln für Standarddeutsch (zumindest die schriftliche Form) seien in der Duden-Grammatik zu finden. Dass sich die unterschiedlichen Duden-Bände nicht immer in der Bewertung einzelner Konstruktionen einig sind, wird den meisten Informant/innen nicht bekannt gewesen sein. Ein Beispiel: In der „Duden-Grammatik“ (2009) findet man wegen im Abschnitt „Präpositionen mit dem Genitiv“, aber mit dem Kommentar: „Die folgenden vier genitivregierenden Präpositionen erlauben, gerade in der gesprochenen Sprache, auch den Dativ: statt, trotz, während, wegen“ (S. 612). Dabei ist es nicht klar, ob „gesprochene Sprache“ auch Standardsprache ist. Im Band „Duden. Basiswissen Schule. Deutsch“ (Duden 2002) steht wegen wieder in der Liste von „Präpositionen mit dem Genitiv“, diesmal aber ist der Kommentar eindeutig präskriptiv: „Umgangssprachlich wird, wegen' oft mit dem Dativ verwendet. Das sollte man vermeiden“ (S. 104). Abonnenten des elektronischen Duden-Newsletters werden auch nicht im Zweifel gelassen: „Welcher Kasus den begehrten Platz hinter der Präposition wegen einnehmen darf, ist vielen klar: In der Standardsprache sollte der Genitiv stehen“ (23.3.2005, archiviert unter www.duden.de). Die Antworten der Lehrenden in den von Davies durchgeführten Umfragen sagen natürlich nur etwas über die Wahrnehmung der Duden-Bände aus und nicht über den tatsächlichen Gebrauch von solchen Werken. Wie auch schon im historischen Überblick oben angesprochen, löst dies nicht unser Problem der mangelnden Erkenntnis darüber, was tatsächlich im Unterricht akzeptiert wird. ${ }^{13}$

12 In vielen deutschsprachigen Werken zur Standardisierung wird die Existenz eines Kodexes als eines der konstitutiven Merkmale einer Standardvarietät angeführt (z.B. Ammon 1995; Löffler 2005). Aus britischer Sicht ist dies nicht so selbstverständlich.

13 Dass nicht immer die tatsächliche Duden-Norm in der Praxis ausschlaggebend ist, zeigen verschiedene Anekdoten. Es wurde W. Davies in Mannheim und Umgebung und M. Wagner in Trier und Umgebung mehr als einmal erzählt, der Duden akzeptierte den Gebrauch von wegen mit Dativ inzwischen als standardsprachlich. Die Vorstellung davon, was im Duden steht, entspricht nicht immer der Wirklichkeit. 


\section{Die heutige Situation: Normendiskussionen}

Wie gesehen, schreiben die Lehrpläne den Gebrauch und die Vermittlung der Standardsprache vor (die Begriffe ,Hochdeutsch' und ,Hochsprache kommen auch vor); es wird aber selten explizit erklärt, was ganz konkret unter diesen Begriffen zu verstehen ist. Sprachwissenschaftliche Diskussionen zu dem Thema finden von Zeit zu Zeit statt, z.B. verlangte Jäger schon 1971: „Zumindest sollte der Spielraum der Norm unter Berücksichtigung der gesprochenen Sprache und der Sprache einer bestimmten Öffentlichkeit erweitert werden“ (Jäger 1971, S. 227). Wagner (1987, S. 134) meint, die gesprochene Standardsprache könne mehr regionale Variation vertragen, ohne dass die gegenseitige Verständigung beeinträchtigt werde: „Auch mit der landschaftlichen Färbung, wie sie eine regionale Ausprägung der Hochsprache bietet, läßt sich eine weiträumige Kommunikation bestreiten." Sieber/Sitta (1986) plädieren dafür, dass sich Lehrerende beim Korrigieren von mündlichen Leistungen eher an „Sprachverständlichkeitsnormen“ als an „Sprachrichtigkeitsnormen“ orientieren sollten. Wir wissen aber nicht genau, ob bzw. in welchem Grad solche Positionen jemals weitflächig den Sprung in den Schulalltag geschafft haben. Dies wird bestimmt nach Lehrer/in, nach Schulart und nach Region variieren. Greulich (1995) berichtet, dass Lehrer/innen an einer Schwetzinger Hauptschule behaupteten, sie würden als lokal empfundene Wörter wie Grumbeere auch in mündlichen Beiträgen von Schüler/innen korrigieren, nicht aber Formen wie ned, die als überregional wahrgenommen wurden.

Macha (1995) beschreibt eine Studie von Grundschullehrer/innen im Rheinland. Laut Fragebogenergebnissen tendierten die Lehrenden dazu, regionale Abweichungen von der Standardaussprache nicht zu korrigieren. Regionale Lexik wurde anscheinend auch toleriert, während grammatische Abweichungen von der Standardsprache (nach statt zu, wie als Vergleichspartikel oder abweichende Kasusrektion) stärker sanktioniert wurden. Was es weder in Deutschland noch in den anderen deutschsprachigen Ländern gegeben hat, ist eine grundsätzliche Kritik der Annahme, dass es sinnvoll ist, die gesprochene Form der Standardsprache zu lehren, ${ }^{14}$ bzw. einen Versuch, die Charakteristika der gesprochenen Standardsprache unabhängig von der geschriebenen Form zu beschreiben und für die Schule zugänglich zu machen (siehe aber Maitz/Elspaß 2013 und Schneider 2013).

Dies ist nicht überall so. In Großbritannien entstand in den 1990er Jahren eine hitzige Debatte zwischen Pädagog/innen, Sprachwissenschaftler/innen, Journalist/innen und Politiker/innen über die konkrete Form des gesprochenen Standardenglischen, die im gerade eingeführten „National

14 Auch die oft als zu radikal kritisierten Hessischen Rahmenrichtlinien Deutsch (1972) erkannten die etablierte Sprachnorm als Lernziel an (siehe Jäger 1974, S. 26). 
Curriculum“ (Lehrplan) für Englisch für den Unterricht vorgeschrieben wurde: Es wurde argumentiert, dass es Lehrenden unmöglich sein würde, diese Sprachform zu vermitteln, da es nicht klar sei, worauf sie sich beziehe. Nicht nur sei sie nicht kodifiziert, sie sei bislang nicht einmal umfassend beschrieben worden. Diese Situation gestaltet sich in Deutschland anders, da durch die einschlägigen Regelwerke ein Kodex der Standardvarietät besteht. ${ }^{15}$ Trotzdem gibt es noch reichlich Grauzonen und Zweifelsfälle, wie der teilweise phänomenale Zuspruch zu den vielen telefonischen und Online-Sprachberatungsstellen, ${ }^{16}$ Sonderwörterbüchern (Sprachunsicherheiten, Zweifelsfälle) und den populärwissenschaftlichen Werken à la Bastian Sick (2004) zeigt. Experten fällt auf, dass sich Widersprüchliches auch in Werken finden lässt, die mit einem wissenschaftlichen Anspruch geschrieben wurden (siehe oben zur Kasusrektion mit wegen). ${ }^{17}$

Dass dies eine ernste Sache ist, zeigen Beispiele, in denen Schüler/innen ihre Note anfechten wollen. Da das Bürgerliche Gesetzbuch keine Aussagen zur Sprachrichtigkeit macht, bedarf es nach Meinung einiger einer entscheidenden Instanz, auf die man sich im Streitfalle berufen kann. Ammon (2003, S. 5-6) führt Beispiele für Fälle an, wo Eltern die Autorität von Lehrkräften anhand des Kodexes in Frage stellten. Außerdem schreibt er, dass das Bundesverwaltungsgericht behaupte, es würde sich auf den Kodex berufen in den seltenen Fällen, wenn Uneinigkeit über den standardsprachlichen Status eines Wortes oder einer Konstruktion zu juristischen Konflikten führt (ebd.). Wonach sollen sich also Lehrer/innen richten, wenn sie sprachliche Richtigkeit und Angemessenheit im Ausdruck der Schülerleistungen bewerten müssen (siehe Vesper 2007, S. 258), wie dies von den Lehrplänen vorausgesetzt wird? Dass dies keineswegs nur theoretische Implikationen hat, zeigt die Reaktion auf die PISA-Ergebnisse in Deutschland, Luxemburg und in der Schweiz, die eine hitzige Debatte über die sprachlichen Fähigkeiten von Schüler/innen und deren Lehrer/innen entfachte.

In unserem letzten Abschnitt diskutieren wir die besondere Dimension des Fremdsprachenunterrichts. Hier gilt vielleicht noch mehr als für den Muttersprachunterricht, dass die Lehrenden gewisse Filter applizieren, um eine möglichst homogene, schrift- oder hochsprachliche Norm als Zielsprache zu vermitteln.

15 Vgl. Klein (in diesem Band) zur Frage, welche Werke Teil des Kodex sind; auch kritisch zur Verortung der Standardsprache und zur Rolle des Kodex: Davies/Langer (2006) und Langer (2007).

16 Man beachte, dass es Ähnliches für das Englische nicht gibt.

17 Pikanterweise werden sogar die Duden-Bände, die wissenschaftlich-deskriptiv geschrieben wurden (z.B. „Duden 4: Die Grammatik“), präskriptiv-autoritativ vermarktet („Duden unentbehrlich für richtiges Deutsch"). 


\section{7. „Richtiges Deutsch“ im Fremdsprachenunterricht}

Vom Fremdsprachenunterricht wird in der Regel erwartet, dass die Student/innen die Standardsprache lernen, in Schrift und Wort. Regionale Varianz wird nicht angestrebt, auch wenn es nach unserer Erfahrung in Großbritannien nur selten vorkommt, dass der Gebrauch von regionalen Varianten unter Studierenden korrigiert wird. Anekdotisch wird uns berichtet, dass einige Studierende ihr Auslandsjahr nicht in Österreich verbringen wollen, weil sie selbst keinen ,Dialekt ${ }^{`}$ lernen wollen, auch wenn dies von Aberystwyth oder Bristol (heutzutage) keinesfalls als negativ bewertet würde. Offizielle Richtlinien hierzu gibt es allerdings fast nie. Die Germanistik am University College London scheint eine Ausnahme zu sein, denn im dortigen „Handbook“ für Studenten heißt es im Abschnitt zur mündlichen Abschlussprüfung: „Regional variation in accent, vocabulary and expression is acceptable" (Langer 2010, S. 405).

Doch solche Toleranz ist nicht überall selbstverständlich. Ransmayr (2006) berichtet, dass bei einem DaF-Lehrgang Lehrmaterial in österreichisch gefärbtem Deutsch von Lektor-Kolleg/innen als für den Unterricht ungeeignet bezeichnet wurde. Die deutschländische Schriftnorm wird weiterhin als die neutralste, prestigeträchtigste, und korrekteste Varietät empfunden:

Ich wage zu behaupten, dass die Annahme, Plurizentrik wäre schon längst ein alter Hut, dass alle deutschsprachigen so wie alle nicht deutschsprachigen Sprachpädagogen heutzutage Bescheid wüssten über das plurizentrische Konzept, es tatsächlich verstanden und verinnerlicht hätten, wohl (noch) eine Illusion ist. In meiner Studie zum Status des österreichischen Deutsch an Germanistik-Instituten an Universitäten in Großbritannien, Frankreich, Ungarn und Tschechien (Ransmayr 2006) zeigte sich, dass vor allem französische und britische Deutsch-Lehrende (und auch Deutsche selber) ein stark monozentrisch geprägtes Bild von der deutschen Sprache haben. (Ransmayr 2012, S. 200)

Auch unter deutschen DaF-Didaktikern gibt es deutliche Unterschiede zu diesem Thema: Zwischen 2001 und 2003 fand in der Zeitschrift „Deutsch als Fremdsprache“ eine Debatte darüber statt, welche Art von Deutsch unterrichtet werden sollte und wie viel Variation die Lernenden vertragen würden. Götze (2001, S. 131) plädierte dafür, ein einheitliches Standarddeutsch zu unterrichten, wie es im Kodex vorgeschrieben werde, da es seiner Meinung nach „verbindlich für alle Teilnehmer der Sprachgemeinschaft" sei. Thurmair (2002, S. 8) zeigt sich toleranter gegenüber sprachlicher Variation, gibt aber nur widerwillig zu, dass es für Lernende nützlich sein könnte, einige typisch sprechsprachliche Formen zu lernen, die von der ,auf dem schriftlichen Sprachgebrauch basierenden Standardnorm“ abweichen würden. DaFLehrer/innen zielen häufig auf eine „variationsfreie Standardsprache“ aus zweierlei Gründen: (1) aus dem praktischen pädagogischen Grund, dass sie 
ihre Studierenden nicht verwirren wollen; (2) aus ideologischen Gründen, d.h. sie hängen an einer Standardideologie und setzen nichtstandardsprachliche Varianten mit ,schlechtem ' Deutsch gleich (Durrell/Langer 2005). Eine auffällige Ausnahme hierzu bilden die für den englischsprachigen DaFUnterricht konzipierten Sprachlehrbücher von Martin Durrell (z.B. Durrell 2003). Er argumentiert für eine register-sensible Sprachlehre, da es für Lernende nicht unbedingt von Nutzen sei, wenn sie ausschließlich die formelle Schriftsprache beherrschten. Sobald sie in einem deutschsprachigen Land ankämen, würden sie regionalen, informellen und gesprochensprachlichen Varianten begegnen (z.B. e-Apokope bei Verben (ich hab), so (ein) statt ein solch, weil + V2, usw.), auf die sie sonst nicht vorbereitet wären. Auch wenn Lernende natürlich nicht jede Form des Deutschen aktiv beherrschen müssen, plädiert er doch dafür, dass

die ausschließliche Vermittlung der herkömmlichen hochsprachlichen Normen heutzutage nicht mehr für die Vermittlung umfassender fremdsprachlicher Kompetenzen ausreicht, denn die Lernenden müssen sich auch die Fähigkeit aneignen, sich an einer Vielfalt sprachlicher Handlungen in angemessener Weise zu beteiligen. (Durrell 2012, S. 103)

Hierzu gehört ein weiterer Aspekt der sprachlichen Variation, den wir bereits oben angesprochen haben und der wenig Beachtung im Klassenzimmer findet: die Plurizentrik. Obwohl z.B. 1986 auf der internationalen Deutschlehrertagung in Bern beschlossen wurde, dass Lehrwerke das Plurizentrizitätsmodell berücksichtigen sollten, um die Sprachwirklichkeit besser wiederzugeben (Clalüna/Fischer/Hirschfeld 2007, S. 45), fehlt ,in Lehr- und Lernmaterialien nach wie vor ein erkennbares linguistisches Konzept hinsichtlich der nationalen Varietäten des Deutschen“" (Hägi 2006, S. 227).

Auch hier gilt es zwischen Wahrnehmung und Unterrichtspraxis zu unterscheiden. In der bereits oben erwähnten Studie von Durrell/Langer (Durrell/Langer 2005; Langer 2010) zeigten sich deutliche Unterschiede der Aussagen der DaF-Lehrenden über die Varietäten, die sie im Unterricht akzeptieren würden, und den konkreten Varianten, die sie in studentischen Arbeiten unkorrigiert lassen würden. Obwohl DaF-Lehrer/innen in Großbritannien und in Irland das Plurizentrizitätsmodell prinzipiell akzeptieren, wussten sie doch oft nicht, welche konkreten Varianten in welchem Land als standardsprachlich gelten. Viele, die behaupteten, sie würden schweizerisches oder österreichisches Hochdeutsch akzeptieren, gaben gleichzeitig an, sie würden Formen wie vergessen auf (A) oder Trottoir (CH) als falsch anstreichen. Aber auch bei bekannteren Varianten (z.B. ist bzw. hat gesessen) ergaben sich große Unterschiede zwischen dem, was die in Großbritannien unterrichtenden Lektor/innen und Dozent/innen als korrekt empfanden. ${ }^{18}$

18 Die Studie besteht aus 77 Fragebögen aus dem Jahre 2004. In ihnen wurden die Informant/ innen gebeten, allgemeine Fragen zur intendierten und akzeptieren Zielvarietät zu beantwor- 
Während der Gebrauch von weil + V2 von allen Befragten für den schriftlichen Sprachgebrauch abgelehnt wurde, wurde die Konstruktion in den mündlichen Beispielsätzen von ca. 50\% Informant/innen nicht korrigiert. Die fehlende Flexion für das schwache Maskulinum wurde für den schriftlichen Gebrauch von 77\% der Informant/innen angemerkt, für den mündlichen aber von nur 34\%. Dies zeigt zwei Dinge: DaFler/innen können sehr wohl zwischen verschiedenen Verwendungsweisen (hier: mündlich - schriftlich) unterscheiden; es zeigt aber auch, dass sich die DaF-Gemeinde keineswegs einig ist, was akzeptabel ist und was nicht. Regionale Varianten wie der am-Progressiv ${ }^{19}$ wurden von nur 60\% der Befragten im schriftlichen Sprachgebrauch korrigiert, Varianten aus der österreichischen Standardsprache wie vergessen auf hingegen von $81 \%$. Insofern spiegelt sich die Kluft zwischen Theorie und Praxis, die wir bezüglich der Standardnorm im Muttersprachunterricht gesehen haben, auch in der Fremdsprachenvermittlung. Dies wird nicht überraschen, sollte aber zu denken geben. Wenn die Lehrenden in ihrer gegebenen, aber auch von ihnen selbst so gesehenen Funktion als Sprachnormvermittler/innen sich nicht in jedem Fall einig sind, ob eine sprachliche Konstruktion Standarddeutsch ist oder nicht, zeigt dies doch mindestens, dass eine allzu enge Definition der Standardsprache nicht der Wirklichkeit entspricht. Wenn selbst die Lehrenden kollektiv - wenn auch nicht individuell - für die Standardsprache eine Reihe von Varianten akzeptieren, scheint es sinnvoll, auch in der Schulpraxis Variation nicht als falsch, sondern als natürlichen Bestandteil der deutschen Sprache, auch der Standardsprache, zu akzeptieren. Wenn man das nicht tut, läuft man Gefahr, eine wirklichkeitsferne Varietät, die nur für die Schule gilt, zu vermitteln. Es stellt sich aber die Frage, woher Lehrende Informationen darüber nehmen sollen, wie elastisch die zu vermittelnden Normen sein sollen und welche Varianten zugelassen werden dürfen.

Oder erübrigen sich alle solche Erwägungen, da die Rolle der Lehrenden als Normautoritäten bzw. Normvermittler/innen in der heutigen Gesellschaft sowieso relativiert werden muss? Knöbls Studie über schwäbische Schulen konstatiert, dass ,[i]m Fall der Schüler [...] die kodifizierten Normen des Standards so gut wie keine Rolle [spielen]. Ihre dominante Sprechlage konstituiert sich aus sprechsprachlich adaptierten Standardformen" (Knöbl 2012, S. 262). Wie diese sprechsprachlichen Standardformen propagiert werden, ist nicht klar, aber die Lehrenden werden nur ein Faktor sein und einer, der weniger wichtig ist, als von Medien und Öffentlichkeit bisher angenommen.

ten und 30 künstliche Beispielsätze mit Varianten zu korrigieren und zu kommentieren (siehe Langer 2010).

19 Laut Auer (2004, S. 83-84) ist diese Form typisch für überregionales gesprochenes Deutsch. 


\section{Literatur}

Adelung, Johann Christoph (1782 [1971]): Umständliches Lehrgebäude der Deutschen Sprache, zur Erläuterung der Deutschen Sprachlehre für Schulen. Leipzig. [Nachdruck Hildesheim/New York 1971.]

Adelung, Johann Christoph (1793): Grammatisch-kritisches Wörterbuch der Hochdeutschen Mundart mit beständiger Vergleichung der übrigen Mundarten, besonders aber der Oberdeutschen. 4 Teile. Leipzig.

Ammon, Ulrich (1995): Die deutsche Sprache in Deutschland, Österreich und der Schweiz. Das Problem der nationalen Varietäten. Berlin/New York.

Ammon, Ulrich (2003): On the social forces that determine what is standard in a language and on conditions of successful implementation. In: Sociolinguistica 17, S. 1-10.

Auer, Peter (2004): Non-standard evidence in syntactic typology: Methodological remarks on the use of dialect data vs spoken language data. In: Kortmann, Bernd (Hg.): Dialectology meets Typology. Dialect grammar from a cross-linguistic perspective. (= Trends in Linguistics: Studies and Monographs 153). Berlin/New York, S. 69-92.

Bahner, Werner/Neumann, Werner (Hg.) (1985): Sprachwissenschaftliche Germanistik. Ihre Herausbildung und Begründung. Berlin.

Beschlüsse der Kultusministerkonferenz: Bildungsstandards im Fach Deutsch für den Mittleren Bildungsabschluss (2003): Internet: www.kmk.org/fileadmin/veroeffentlichun gen_beschluesse/2003/2003_12_04-BS-Deutsch-MS.pdf (Stand: 6.3.2013).

Bluhm-Faust, Claudia (2005): Die Pädagogisierung der deutschen Standardsprache im 19. Jahrhundert am Beispiel Badens. (= VarioLingua 25). Frankfurt a.M.

Burgwardt, Heinrich (1867): Der Bildungsfreund. 1. Theil: Ein vaterländisches SchulLesebuch zunächst für Mecklenburg. Altona.

Clalüna, Monika/Fischer, Roland/Hirschfeld, Ursula (2007): Alles unter einem D-A-CH-L? Oder: wie viel Plurizentrik verträgt ein Lehrwerk? In: Fremdsprache Deutsch 37, S. 38-45.

Davies, Winifred V. (1995): Linguistic variation and language attitudes in MannheimNeckarau. (= Zeitschrift für Dialektologie und Linguistik, Beihefte 91). Stuttgart.

Davies, Winifred V. (2000): Linguistic norms at school. A survey of secondary-school teachers in a central German dialect area. In: Zeitschrift für Dialektologie und Linguistik 67, S. 129-147.

Davies, Winifred V. (2005): Deutschlehrer und Deutschlehrerinnen (in Deutschland) als Geber und Vermittler von sprachlichen Normen. In: Roggausch (Hg.), S. 323-339.

Davies, Winifred V./Langer, Nils (2006): The making of bad language. Lay linguistic stigmatisations in German: past and present. (= VarioLingua 28). Frankfurt a.M. u.a.

Duden (2002): Duden. Basiswissen Schule. Deutsch. Mannheim u.a. 
Duden (2009): Der Duden. Bd. 4: Die Grammatik. Unentbehrlich für richtiges Deutsch. 8., überarb. Aufl. Hrsg. v. d. Dudenredaktion. Mannheim u.a.

Durrell, Martin (2003): Using German. A guide to contemporary usage. 2. Aufl. Cambridge.

Durrell, Martin (2007): „Deutsch ist eine würde-lose Sprache“. On the history of a failed prescription. In: Elspaß, Stephan et al. (Hg.): Germanic language histories „from Below" (1700-2000). (= Studia linguistica Germanica 86). Berlin/New York, S. 243258.

Durrell, Martin (2012): Zur Relativierung von hochsprachlichen Normen in der deutschen Sprache der Gegenwart. Der Blick von außen. In: Günthner, Susanne et al. (Hg.): Kommunikation und Öffentlichkeit: Sprachwissenschaftliche Potenziale zwischen Empirie und Norm. (= Reihe Germanistische Linguistik 296). Berlin, S. $89-105$.

Durrell, Martin/Langer, Nils (2005): Gutes Deutsch und schlechtes Deutsch an britischen und irischen Hochschulen. Zur Akzeptanz von Variation im DaF-Unterricht. In: Roggausch (Hg.), S. 297-314.

Eichinger, Ludwig M. (2005): Standardnorm, Sprachkultur und die Veränderung der normativen Erwartungen. In: Eichinger/Kallmeyer (Hg.), S. 363-381.

Eichinger, Ludwig M./Kallmeyer, Werner (Hg.) (2005): Standardvariation: Wie viel Variation verträgt die deutsche Sprache? (= Jahrbuch des Instituts für Deutsche Sprache 2004). Berlin/New York.

Eichinger, Ludwig M. et al. (2009): Aktuelle Spracheinstellungen in Deutschland. Mannheim.

Gardt, Andreas (1999): Geschichte der Sprachwissenschaft in Deutschland. Vom Mittelalter bis ins 20. Jahrhundert. Berlin/New York.

Götze, Lutz (2001): Normen - Sprachnormen - Normtoleranz. In: Deutsch als Fremdsprache 38, S. 131-133.

Greulich, S. (1995): Die Verwendung von Dialekt und Hochsprache im Deutschunterricht. Wissenschaftliche Hausarbeit. PH Heidelberg.

Groth, Klaus (1852): Quickborn. Volksleben in plattdeutschen Gedichten ditmarscher Mundart. Hamburg.

Hägi, Sara (2006): Nationale Varietäten im Unterricht Deutsch als Fremdsprache. (= Duisburger Arbeiten zur Sprach- und Kulturwissenschaft 64). Frankfurt a.M. u.a.

Hemmer, Jakob (1769): Abhandlung über die deutsche Sprache zum Nutzen der Pfalz. Mannheim.

Heynatz, Johann Friedrich (1777): Deutsche Sprachlehre zum Gebrauch der Schulen. 3., verm. u. verb. Aufl. Berlin.

Hildebrand, Rudolf (1867): Vom deutschen Sprachunterricht in der Schule und von deutscher Erziehung und Bildung überhaupt. Leipzig. 
Hirt, Herman (1925): Geschichte der deutschen Sprache. 2., neubearb. Aufl. (= Handbuch des deutschen Unterrichts an höheren Schulen 4, Teil 1). München.

Hundt, Markus (2000): „Spracharbeit“ im 17. Jahrhundert. Studien zu Georg Philipp Harsdörffer, Justus Georg Schottelius und Christian Gueintz. (= Studia linguistica Germanica 57). Berlin/New York.

Illgner, Gerhard (2001): Die deutsche Sprachverwirrung. Lächerlich und ärgerlich: Das neue Kauderwelsch. 2. Aufl. Paderborn.

Jäger, Siegfried (1971): Sprachnorm und Schülersprache. In: Sprache und Gesellschaft. Beiträge zur soziolinguistischen Beschreibung der deutschen Gegenwartssprache. Jahrbuch des Instituts für Deutsche Sprache 1970. (= Sprache der Gegenwart 13). Düsseldorf, S. 166-233.

Jäger, Siegfried (1974): Die Hessischen Rahmenrichtlinien „Deutsch“ und die Sprachrichtigkeit. Der Übergang von einer willkürlich-formal-normativen zu einer funktionalen Auffassung. In: Germanistische Linguistik 74/1-2, S. 21-35.

Kernlehrplan für den verkürzten Bildungsgang des Gymnasiums Sekundarstufe I (G8) in Nordrhein-Westfalen. Deutsch (2007). (= Schule in NRW 3409). Frechen.

Knees, Friedrich (Hg.) (1878): Fibel, oder naturgemäßer Lerngang im Rechtschreiben und Rechtlesen. (Schreibendlesen - buchstabiren - lautiren). 15. Aufl. Kiel.

Knöbl, Ralf (2012): Dialekt - Standard - Variation. Formen und Funktionen von Sprachvariation in einer mittelschwäbischen Schulklasse. (= OraLingua 1). Heidelberg.

Kromayer, Johannes (1618 [1986]): Deutsche Grammatica zum newen Methodo der Jugend zum besten zugerichtet. [Nachdruck Hildesheim 1986.]

Langer, Nils (2001): Linguistic purism in action. How auxiliary tun was stigmatised in Early New High German. (= Studia linguistica Germanica 60). Berlin/New York.

Langer, Nils (2004): Frühe Fremdsprachengrammatiken als Vermittlerinnen der Standardsprache. In: Mattheier, Klaus J./Nitta, Haruo (Hg.): Sprachwandel und Gesellschaftswandel - Wurzeln des heutigen Deutsch. München, S. 223-243.

Langer, Nils (2007): Locating Standard German. On the difference between a codex in the head and a codex on the bookshelf. In: Fandrych, Christian/Salvierda, Reinier (Hg.): Standard, Variation and Language Contact in the Germanic Languages. Tübingen, S. 217-241.

Langer, Nils (2010): Sprechereinstellungen zur Zielsprache im britischen und irischen DaF-Unterricht. In: Anders, Christina Ada et al. (Hg.): „Perceptual Dialectology“: Neue Wege der Dialektologie. (= Linguistik - Impulse \& Tendenzen 38). Berlin/ New York, S. 409-431.

Langer, Nils (2011): Historical Sociolinguistics in Nineteenth-Century Schleswig Holstein: Evidence from School Inspection Reports. In: German Life and Letters 64, S. $167-187$.

Langer, Nils/Langhanke, Robert (2013): How to deal with non-dominant languages metalinguistic discourses on Low German in the Nineteenth Century. In: Linguistik-online 58, S. 77-97. 
Law, Claudia (2007): Sprachratgeber und Stillehren in Deutschland (1923-1967): Ein Vergleich der Sprach- und Stilauffassung in vier politischen Systemen. (= Studia linguistica Germanica 84). Berlin/New York.

Lehrplan Deutsch für das Gymnasium in Sachsen (2004). Dresden.

Lippi-Green, Rosina (2012): English with an accent. Language, ideology and discrimination in the United States. 2. Aufl. London/New York.

Löffler, Heinrich (2005): Wie viel Variation verträgt die deutsche Standardsprache? Begriffsklärung: Standard und Gegenbegriffe. In: Eichinger/Kallmeyer (Hg.), S. 7-27.

Macha, Jürgen (1995): Regionalsprachlichkeit und Korrektur in der Grundschule. In: Der Deutschunterricht 47, S. 78-83.

McLelland, Nicola (2012): J.G. Schottelius's Ausführliche Arbeit von der Teutschen Haubtsprache (1663) and its place in early modern European vernacular language study. Oxford.

Maitz, Péter/Elspaß, Stephan (2007): Warum der „Zwiebelfisch“ nicht in den Deutschunterricht gehört. In: Info DaF 34, S. 515-526.

Maitz, Péter/Elspaß, Stephan (2011): „Dialektfreies Sprechen - leicht gemacht!“ Sprachliche Diskriminierung von deutschen Muttersprachlern in Deutschland. In: Der Deutschunterricht 63, 6, S. 7-17.

Maitz, Péter/Elspaß, Stephan (2013): Zur Ideologie des „Gesprochenen Standarddeutsch“. In: Hagemann, Jörg/Klein, Wolf Peter/Staffeldt, Sven (Hg.): Pragmatischer Standard. (= Stauffenburg Linguistik 73). Tübingen.

Müller, Johannes (1882 [1969]): Quellenschriften und Geschichte des deutschsprachlichen Unterrichtes bis zur Mitte des 16. Jahrhunderts. Gotha. [= Nachdruck 1969, mit einer Einführung von Monika Rössing-Hager. Darmstadt].

Naumann, Bernd (1986): Grammatik der deutschen Sprache zwischen 1781 und 1856. (= Philologische Studien und Quellen 114). Berlin.

Rahmenplan Deutsch für das neunstufige Gymnasium in Hamburg (2003). Hamburg.

Rahmenrichtlinien Deutsch für das Gymnasium Schuljahrgänge 5-12 in Sachsen-Anhalt (2003). Magdeburg.

Ransmayr, Jutta (2006): Der Status des österreichischen Deutsch an nichtdeutschsprachigen Universitäten: eine empirische Untersuchung. (= Österreichisches Deutsch Sprache der Gegenwart 8). Frankfurt a.M. u.a.

Ransmayr, Jutta (2012): Zur Wahrnehmung der Varietäten des Deutschen im Unterricht für Deutsch als Muttersprache und Deutsch als Fremdsprache. In: Der Sprachdienst 56, S. 198-207.

Raumer, Rudolf von (1852): Der Unterricht im Deutschen. In: Raumer, Karl von (Hg.): Geschichte der Pädagogik: vom Wiederaufblühen klassischer Studien bis auf unsere Zeit. Theil 3, Abth. 2. 2., unveränd. Aufl. Stuttgart, S. 17-151.

Richtlinien und Lehrpläne für die Sekundarstufe II Gymnasium/Gesamtschule in Nordrhein-Westfalen (1999). Frechen. 
Roggausch, Werner (Hg.) (2005): Germanistentreffen: Deutschland - Großbritannien Irland. Bonn.

Schmidt, Gunther (1985): Sprachkonzeptionen und Schulpraxis. Bemerkungen zu sprachtheoretischen Grundlagen des Deutschunterrichts. In: Bahner/Neumann (Hg.), S. 249-281.

Schmidt, Hartmut (1985): Aspekte der Institutionalisierung. Zur Durchsetzung der neuen Denkmuster. In: Bahner/Neumann (Hg.), S. 151-248.

Schneider, Jan-Georg (2013): Sprachliche ,Fehler ${ }^{6}$ aus sprachwissenschaftlicher Sicht. In: Sprachreport 29, 1-2, S. 30-37.

Schneider, Wolf (2001): Deutsch fürs Leben. Was die Schule zu lehren vergaß. 10. Aufl. Reinbek bei Hamburg.

Schneider, Wolf (2012): Die Sprache ist eine Waffe. Aber sie lässt uns auch träumen, sie erklärt uns die Welt. Seien wir gut zu ihr! In: Die Zeit online 11.5.2012. Internet: www.zeit.de/2012/20/Sprache (Stand: 26.2.2013).

Schneider-Mizony, Odile (2008): Grammatiken der wilhelminischen Ära: Dokumente der deutschen Sprache oder Monumente der Norm? In: Lartillot, Françoise/Gellhaus, Axel (Hg): Dokument - Monument. Textvarianz in den verschiedenen Disziplinen der europäischen Germanistik. (= Convergences 46). Bern, S. 395-410.

Sick, Bastian (2004): Der Dativ ist dem Genitiv sein Tod. Ein Wegweiser durch den Irrgarten der deutschen Sprache. Köln.

Sieber, Peter/Sitta, Horst (1986): Mundart und Standardsprache als Problem der Schule. (= Sprachlandschaft 3). Aarau u.a.

Siegl, Elke A. (1986): Der Duden als Normierungsinstrument in der DDR und in der Bundesrepublik Deutschland. In: Debus, Friedhelm et al. (Hg.): Sprachliche Normen und Normierungsfolgen in der DDR. (= Germanistische Linguistik 82/83). Hildesheim u.a., S. 75-88.

Stickel, Gerhard/Volz, Norbert (1999): Meinungen und Einstellungen zur deutschen Sprache. Ergebnisse einer bundesweiten Repräsentativerhebung. (= amades 2/1999). Mannheim.

Thurmair, Maria (2002): Standardnorm und Abweichungen. Entwicklungstendenzen unter dem Einfluss der gesprochenen Sprache. In: Deutsch als Fremdsprache 39, S. 3-8.

Vesper, Wilhelm (2007): Ist gutes Deutsch heutzutage schon das weniger schlechte Deutsch? Einige Überlegungen zu der Frage nach dem guten Deutsch im schulischen Unterricht. In: Burkhardt, Armin (Hg.): Was ist gutes Deutsch? Studien und Meinungen zum gepflegten Sprachgebrauch. (= Thema Deutsch 8). Mannheim u.a., S. $255-264$.

Volmert, Johannes (1997): Jugend und Ruhrgebietssprache: Die regionale Varietät in der Freizeit - und als Unterrichtsgegenstand? In: Ehlich, Konrad et al. (Hg.): Sprache und Literatur an der Ruhr. 2., erw. u. überarb. Aufl. (= Schriften des FritzHüser-Instituts für Deutsche und Ausländische Arbeiterliteratur der Stadt Dortmund: Reihe 2, Forschungen zur Arbeiterliteratur 10). Essen, S. 57-79. 
Wagner, Eberhard (1987): Das fränkische Dialektbuch. München.

Wagner, Melanie (2009): Lay linguistics and school teaching: An empirical sociolinguistic study in the Moselle-Franconian dialect area. (= Zeitschrift für Dialektologie und Linguistik, Beihefte 136). Stuttgart.

Wienbarg, Ludolf (1834): Soll die plattdeutsche Sprache gepflegt oder ausgerottet werden? Gegen Ersteres und für Letzteres. Hamburg.

Wustmann, Gustav (1966): Allerhand Sprachdummheiten. Kleine deutsche Grammatik des Zweifelhaften, des Falschen und des Häßlichen. Ein Hilfsbuch für alle, die sich öffentlich der deutschen Sprache bedienen. 14., ern. Aufl. Berlin.

Ziegler, Evelyn (1998): Zur Entwicklung der Standardsprache und der Vorbildfunktion der Klassikersprache. In: Der Deutschunterricht 3, S. 24-31. 
